\title{
La empleabilidad de los egresados de las universidades tecnológicas en México: un análisis de eficiencia
}

\author{
José-Gabriel Aguilar-Barceló y Ana-Bárbara Mungaray-Moctezuma
}

\section{RESUMEN}

El sistema de universidades tecnológicas enfrenta importantes retos para atender con calidad la demanda de trabajo técnico especializado. A través del Análisis Envolvente de Datos, se evalúa la eficiencia de este sistema en materia de empleabilidad. Se encuentra que la eficiencia técnica más alta está en la región norte, y la más baja, en la sur. Todas las regiones presentan dificultades referidas a la pertinencia, vinculación o equidad. Se concluye que el desempeño ha sido desigual entre universidades pero también entre regiones. Lo anterior sería muestra de que los distintos planteles no tienen las mismas oportunidades y criterios de decisión en la búsqueda de objetivos comunes.

Palabras clave: universidades tecnológicas, evaluación institucional, indicadores educativos, eficiencia académica, educación y empleo.

José-Gabriel Aguilar-Barceló

gaba@uabc.edu.mx

Mexicano. Doctor en Ciencias Económicas por la Universidad Autónoma de Baja California (UABC), México. Profesor e investigador de la Facultad de Economía y Relaciones Internacionales, UABC, México. Miembro del Sistema Nacional de Investigadores nivel II. Principales temas de investigación: economía del emprendimiento, desarrollo social y medición de la eficiencia.

\section{Ana-Bárbara Mungaray-Moctezuma}




\section{A empregabilidade dos recém-formados das universidades tecnológicas no México: uma análise de eficiência}

\section{RESUMO}

O sistema de universidades tecnológicas enfrenta importantes desafios para atender com qualidade a demanda de trabalho técnico especializado. Através da Análise Envolvente de Dados, se avalia a eficiência deste sistema para a empregabilidade. Se nota que a eficiência técnica mais alta está na região norte, e a mais baixa, na sul. Todas as regiões apresentam dificuldades referidas à pertinência, vinculação ou equidade. Se conclui que o desempenho tem sido desigual entre universidades e também entre regiões. $\mathrm{O}$ anterior seria exemplo de que os diferentes grupos de recém-formados não têm as mesmas oportunidades e critérios de decisão na busca de objetivos comuns.

Palavras chave: universidades tecnológicas, avaliação institucional, indicadores educativos, eficiência acadêmica, educação e emprego.

\section{The Employability of Graduates of Technological Universities in Mexico: An Analysis of Efficiency}

\section{ABSTRACT}

The system of technological universities faces important challenges to meet with quality the demand for specialized technical work. Through the Enveloping Data Analysis, the efficiency of this system is evaluated in terms of employability. The highest technical efficiency is found in the northern region, and the lowest in the south. All regions have difficulties related to relevance, linkage or equity. It is concluded that performance has been unequal among universities but also among regions. This would show that the different campuses do not have the same opportunities and decision-making criteria in the search for common objectives.

Key words: technological universities, institutional evaluation, educational indicators, academic efficiency, education and employment. 


\section{Introducción}

En 1991 surgen en México las universidades tecnológicas (UT) con el objetivo de generar una vía de solución a las demandas de trabajo técnico especializado (Flores-Crespo, 2009), preparar recursos humanos con conocimientos y habilidades basados en la ciencia, y construir una capacidad tecnológica que sustentara lo que se advertía como un promisorio proceso industrializador (Ruiz-Larraguivel, 2011). No obstante, en la actualidad, el sistema de universidades tecnológicas enfrenta importantes desafíos relacionados con su potencial para satisfacer con calidad y oportunidad, la demanda de trabajo técnico especializado y generar el capital humano tecnológico que soporte el proceso industrializador del país.

La oferta educativa de las UT en México se basa fundamentalmente en carreras de Técnico Superior Universitario (TSU), en áreas como la manufactura, agroindustria, técnicas de información y comunicación (TIC), gestión administrativa, química y servicios, cuyo plan de estudios se imparte en un periodo de dos años. ${ }^{1}$ Figuras como esta existen en muchos países del mundo entre los que destacan Francia (Institute Universitarie de Technologie), Japón (Semangokko), Alemania (Fachoschülen), Estados Unidos y Canadá (Community College) (Villa, 2008).

Aunque el término "empleabilidad" finalmente se asocia a las posibilidades que tiene un profesional de encontrar empleo, también hace referencia al desarrollo de elementos como los conocimientos, experiencias, habilidades y actitudes necesarias para alcanzar una alta productividad en un mercado laboral que se presenta cada vez más competido. Estos elementos deben ajustarse en función de las necesidades del mercado de trabajo; su nivel, en buena medida, es responsabilidad de las instituciones educativas por las que pasa el potencial profesionista. De lo anterior se deriva la importancia de evaluar la operación de estas instituciones en términos del uso de los insumos con los que cuentan para conseguir sus objetivos. Lejos de ser un proceso meramente técnico, la evaluación educativa es un mecanismo que debe estar vinculado directamente al tipo de proyecto productivo, económico o social que se busca implantar o consolidar (González, 2001).

Con el apoyo de la metodología de análisis envolvente de datos, en el presente trabajo se pretende conocer cómo se relacionan los resultados de la operación de las UT con sus insumos productivos, y cuáles de éstos están más asociados con sus metas de empleabilidad y formación continua, y, al mismo tiempo, son mejor aprovechados en la consecución de éstas. Además, el análisis estadístico, permite describir el comportamiento regional de este tipo de universidades en México e identificar algunas de las características que hacen que unas de sus sedes sean más eficientes que otras en el marco de la región geográfica a la que pertenecen y en términos de la satisfacción de egresados y empleadores.

\section{Problemática de las universidades tecnológicas}

El modelo educativo de las UT se sustenta en cinco atributos que orientan su quehacer académico: pertinencia, intensidad, continuidad, polivalencia y flexibilidad (GGUT-SEP, 2004). De acuerdo con Flores-Crespo (2009), la pertinencia se refiere a la correspondencia entre su operación y las expectativas de la población. El aprovechamiento del tiempo, esfuerzo y recursos durante el proceso de enseñanza-aprendizaje describen el nivel de intensidad, mientras que la continuidad implica que los estudiantes pueden seguir con sus estudios en otras instituciones educativas de así requerirlo. Además, la

\footnotetext{
${ }^{1}$ Desde 2009 se pueden estudiar también licenciaturas (en tres años) y especialidades tecnológicas (en cuatro años).
} 
polivalencia significa que los estudiantes adquieren los conocimientos y habilidades con la suficiente versatilidad como para trabajar en las diferentes fases de un proceso productivo, adoptando las tecnologías que ello demande (Villa, 2008). Por último, la flexibilidad se refiere a que los planes de estudio se adaptan continuamente, según las necesidades del sector productivo, estando asociado este concepto también con las facilidades que tiene el estudiante para adquirir una formación multidisciplinaria (GGUT-SEP, 2004).

No obstante que los esfuerzos han ido encaminados a fortalecer estos atributos, el sistema de UT presenta una sintomatología a la que habría que prestar atención. En términos de su evolución y de acuerdo con estimaciones propias utilizando datos de García (2009), las UT no han podido sostener el ritmo de crecimiento que precedió a su fundación, habiendo atendido no más del $4 \%$ de la matrícula en educación superior para el año 2010. Además, aunque las UT no están diseñadas para albergar grupos numerosos (Ruiz-Larraguivel, 2011), muchos planteles no llegan a 1000 estudiantes, lo que es equivalente a mantener una capacidad ociosa promedio del $35 \%$ de la infraestructura, que en algunos casos supera el 50\% (López, 2008).

Por otra parte, existen problemas asociados a la pertinencia y vinculación de los programas académicos que están ocasionando que la figura del egresado de la UT no sea adecuadamente valorada por la industria (Silva, 2006). Hay una falta de reconocimiento por parte de las empresas acerca de la calificación ocupacional del TSU, lo que se ha traducido en dificultades de inserción al mercado de trabajo y de movilidad laboral ascendente (Ruiz, 2009; Ruiz-Larraguivel, 2011). De hecho, no suele existir dentro de la estructura orgánica de las empresas un nivel jerárquico que ubique al TSU dentro de la organización (López, 2008). También, en materia académica, la capacidad analítica, lógica y de razonamiento abstracto de los egresados, no ha estado al nivel que el sector productivo espera (Silva, 2006).
Finalmente, en opinión de los empresarios, el TSU no representa una ventaja competitiva sobre otros profesionales en términos de capacidad analítica y liderazgo (Villa, 2008).

Aunque debe reconocerse que las UT han ayudado a generar una diversidad necesaria en la oferta educativa, autores como Silva (2006) afirman que las UT, al demandar hasta el $80 \%$ del tiempo que requiere una licenciatura - hecho que no se compensa con la expectativa salarial-, resultan poco atractivas. Para López (2008), este culto a la escolarización es muestra de que las UT no están compatibilizando la aspiración de estudiar, con la necesidad de trabajar o de adquirir experiencia laboral, lo cual restringe las oportunidades de los prospectos poniéndolos en relativa desventaja económica y social.

Como consecuencia de lo anterior, cabría preguntarse si las UT están cubriendo las expectativas de los egresados, pero sobre todo, si éstos están satisfaciendo las necesidades que el sector productivo demanda. En este constructo de calidad educativa, sin duda debe considerarse la eficiencia en la asignación y aprovechamiento de los recursos con los que se cuenta para conseguir los objetivos institucionales (Valenzuela et al., 2009), donde el desempeño de los egresados toma una importancia estratégica. De hecho, para Mazeran et al. (2006), el éxito de una institución de educación superior, más allá de reconocimientos y acreditaciones, se debe medir por los resultados de sus egresados.

\section{Evaluación de las instituciones educativas}

Las instituciones públicas sin ánimo de lucro no son ajenas a la necesidad de conocer si la asignación de recursos que llevan a cabo es la adecuada para conseguir sus fines, aunque éstos no sean de carácter económico sino social. En este contexto, por ejemplo, Martínez y Guzmán (2014) evalúan el rendimiento de las entidades sin fines de lucro en España, encontrando que las buenas prácticas están asociadas a una sana combinación entre liquidez y 
endeudamiento, mientras que el tamaño y la antigüedad de la institución la alejan de esta frontera.

Aunque en materia de evaluación educativa se ha escrito profusamente durante varias décadas, la literatura acerca de la evaluación de la eficiencia de las unidades de servicios educativos no empezó a ser prolífica hasta el inicio de este siglo. Entre los trabajos más destacados en este campo está el de Johnes (2006), quien encuentra que el gasto administrativo, el número de egresados y el de aquellos que dan continuidad a sus estudios, son insumos relevantes en la determinación de la eficiencia, mientras que el número y calidad de los programas disponibles y la investigación asociada a estos, pueden ser considerados como productos importantes en esta medición. ${ }^{2}$

De acuerdo con Lenin et al. (2016), los estudios que evalúan la enseñanza de las universidades consideran aspectos relacionados con el financiamiento como parte de los insumos, mientras que, en la conformación de las salidas, no es extraño encontrar variables como la matrícula y el número de titulados de licenciatura y posgrado, el total de docentes y el número de sus publicaciones. En el campo de la medición de la eficiencia en los departamentos universitarios en ciertas regiones de España, Martín (2008) encuentra que es posible mejorar la gestión siempre que se consiga una asignación de recursos más pertinente en materia educación superior. Por su parte, Bradley et al. (2010) afirman que variables como el género, la edad y la etnicidad de los estudiantes pueden llegar a ser más importantes que aquellas relacionadas con el personal universitario, en la determinación del nivel de eficiencia de las instituciones de educación superior inglesas.

Específicamente en el ámbito de la educación técnico-científica, Lucena y Moreira (2015) estudian los resultados de los institutos federales de educación, Ciencia y Tecnología en Brasil y su relación con los costos y posibilidades de expansión. Encuentran que menos de un tercio de los institutos es eficiente; el trabajo mostró, además, que los mejores resultados en términos de titulación no estaban vinculados a un mayor gasto por alumno. Por otro lado, Stukalina (2012) encuentra que en la toma de decisiones relacionada con las mejoras en la calidad de la educación técnica es importante incluir el grado de satisfacción percibida de los estudiantes, así como sus motivaciones, surgidas de la interacción con el entorno.

Nazarko y Saparauskas (2014) evalúan un conjunto de universidades tecnológicas polacas, encontrando que las variables ambientales y contextuales, además de las convencionales variables de insumo y de producto, deben ser consideradas en los análisis. Además, de acuerdo con Ghose (2017), incluir los efectos de becas, subsidios, instalaciones e indicadores sociales (como aquellos vinculados a la desigualdad y la pobreza) y de contexto político podría contribuir al logro de resultados más precisos en los estudios de eficiencia de las instituciones de servicios educativos. ${ }^{3}$ Todos los trabajos anteriores se apoyan del Análisis Envolvente de Datos (AED) como herramienta de análisis.

Concretamente para las UT mexicanas, aunque la Misión Internacional de Evaluación Externa de las UT aseguró en su momento que el subsistema constituía una inversión pública exitosa al haber formado profesionales eficaces en su desempeño laboral y promovido el desarrollo local y regional (Mazeran et al., 2006), Silva (2008), en lo referente a la atención integral al estudiante y los proceso centrados en el aprendizaje, advierte la ausencia de indicadores eficaces para medir los resultados de las acciones dirigidas a transformar los procesos sujetos a mejora.

Por su parte, Valenzuela et al. (2009) determinan las características que debe tener un modelo de evaluación que ayude a mejorar la gestión de

${ }^{2}$ Cabe aclarar que en este trabajo se utiliza el término "entrada" o "insumo" en lugar del anglosajón input; asimismo se utiliza el término "salida", "producto" o "resultado" por el de output.

${ }^{3}$ Aunque podría generalizase para la eficiencia de cualquier nivel educativo, esta afirmación está referida al entorno de la educación básica. 
las instituciones educativas y la calidad de los servicios que ofrecen. Su modelo institucional destaca el peso de indicadores relacionados con la formación integral, el capital humano, el prestigio social y la sustentabilidad económica, entre otros. El tema es relevante pues en un estudio sobre la eficacia educativa de las UT, Hernández-González et al. (2008), controlando el factor socioeconómico, encontraron que sólo $16 \%$ de las universidades generaba un valor agregado significativo sobre sus alumnos; para ello utilizaron indicadores asociados a las diferencias de desempeño de los exámenes de ingreso y egreso.

Lo descrito en esta sección da muestra de la complejidad asociada a la determinación de los conjuntos de entradas y salidas para la medición de la eficiencia, existiendo incluso indicadores que pueden ser considerados insumo para una investigación y producto para otra, de acuerdo con las metas institucionales, el entendimiento de la dinámica y proceso educativo por parte de los directivos y la disponibilidad de información.

\section{Metodología y datos Población y muestra}

Debido a que las condiciones demográficas, ocupacionales y sociales no son iguales a lo largo del país, en este trabajo las UT se han clasificado por regiones, favoreciendo así la interpretación de los resultados estadísticos debido a la ganancia en homogeneidad de las unidades de medición. Con la intención de simplificar el análisis, las entidades federativas del país fueron conglomeradas geográficamente con base en Fouquet (2002), para quedar como se ve en la figura 1: región norte (compuesta por las zonas económicas noroeste y noreste), región sur (compuesta por las zonas económicas suroeste y sureste), región centro-oeste (conformada por las zonas económicas oeste y centro-norte) y región centro-este (que considera las zonas económicas este y centro-sur). ${ }^{4}$

\section{Figura 1. Mapa de México por entidades federativas y regiones}

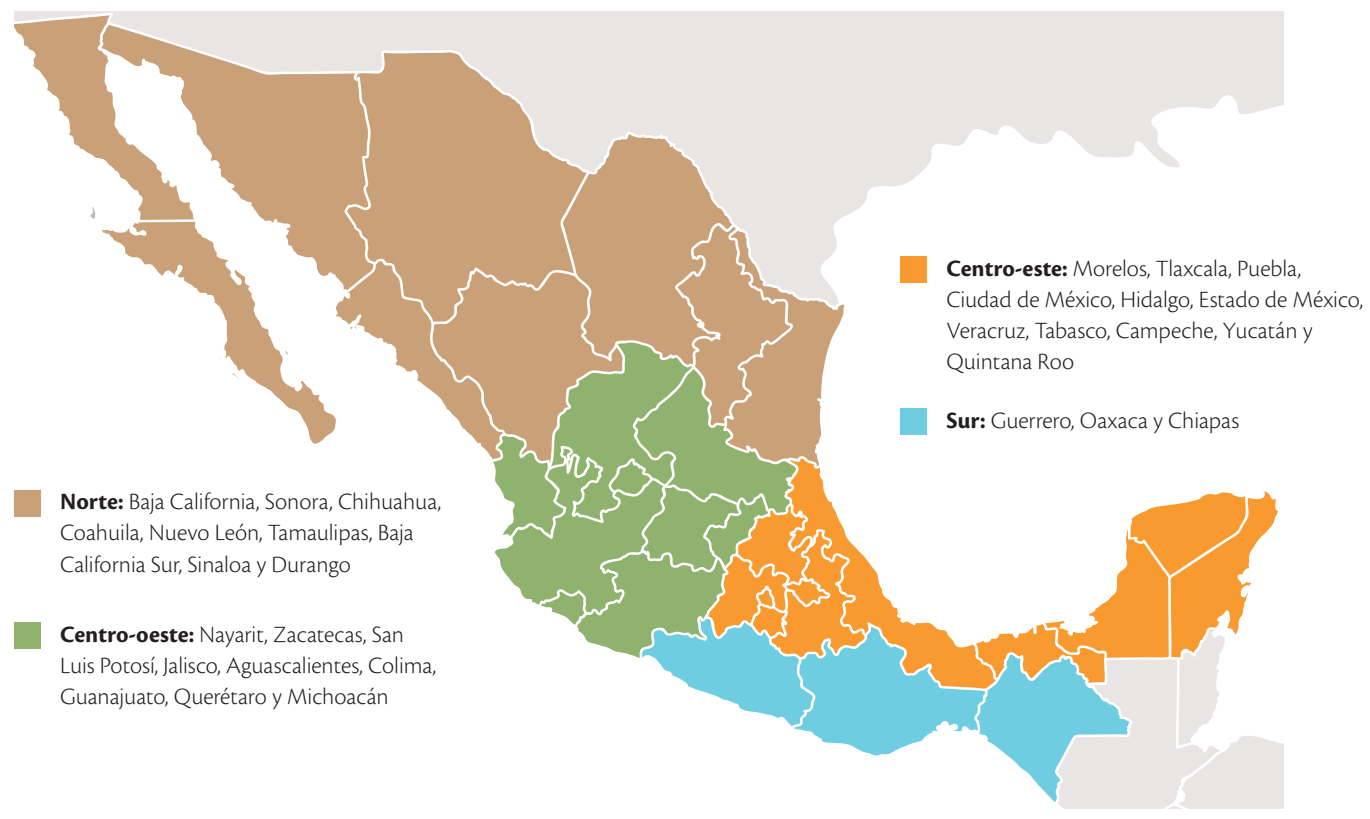

Fuente: elaboración propia con apoyo de http://www.paintmaps.com

${ }^{4}$ Existen otras clasificaciones como la referida a las regiones socioeconómicas propuesta por el Instituto Nacional de Estadística y Geografia; sin embargo, ésta no proporciona suficientes observaciones en cada categoría, lo cual era indispensable para el tratamiento estadístico de la información, más allá de que presenta dispersión geográfica que trae consigo inconvenientes argumentativos sobre el desarrollo regional. 
La información utilizada y de dónde se obtienen todos los indicadores que constituyen el instrumento de investigación procede de la base de datos MECASUT de 2014 (la más reciente disponible en el momento de realizar este trabajo) que a su vez se basa en el modelo de evaluación de la calidad del Subsistema de Universidades Tecnológicas (MECASUT, 2011). La investigación considera 62 de las 109 UT con las que contaba el sistema en el citado año. Cabe señalar que se han descartado las UT con vacíos en más de un tercio de los campos ante la duda sobre la veracidad de la información o la sospecha de falta de participación en los procesos sustanciales que rigen su quehacer. También han quedado fuera del análisis las universidades con matrícula inferior a 1000 estudiantes por estar asociadas con zonas rurales, limitada oferta académica o áreas de especialización, lo que en todo caso introduce heterogeneidad en el estudio. La tabla 1 enumera las UT consideradas y su clasificación.

Tabla 1. Universidades Tecnológicas consideradas en la investigación

\begin{tabular}{|c|c|c|c|}
\hline Región norte & Región sur & Región centro-oeste & Región centro-este \\
\hline Hermosillo & Costa-Grande-Gro. & Aguascalientes & Nezahualcóyotl \\
\hline Nogales & Selva & Norte-Guanajuato & Fidel-Velázquez \\
\hline Tijuana & Región-Norte-Gro. & Querétaro & Tecámac \\
\hline Ciudad-Juárez & Valles-Centrales-Oax. & León & Sur-Edo.-México \\
\hline Chihuahua & Tabasco & San-Luis-Potosí & "E.-Zap."-Mor. \\
\hline Sur-Sonora & Campeche & Zacatecas & Valle-Toluca \\
\hline Durango & Cancún & Suroeste-Guanajuato & Tula-Tepeji \\
\hline Coahuila & San-Juan-del-Río & Norte-Aguascalientes & Puebla \\
\hline Izúcar-Matamoros & Metropolitana & Salamanca & Tulancingo \\
\hline Gral-Mariano-Escobedo & & Jalisco & Huasteca-Hidalguense \\
\hline Norte-Coahuila & & Morelia & Tecamachalco \\
\hline Santa-Catarina & & Nayarit & Tlaxcala \\
\hline Torreón & & Costa-Nayarit & Valle-Mezquital \\
\hline Tamaulipas-Norte & & Zona-Metrop.-Gdl. & Sierra-Hidalguense \\
\hline Matamoros & & Bahía Banderas & Huejotzingo \\
\hline Región-Centro-Coahuila & & & Xicotepec-Juárez \\
\hline Altamira & & & Sureste-Veracruz \\
\hline Nuevo-Laredo & & & Centro-Veracruz \\
\hline Cadereyta & & & Gutiérrez-Zamora \\
\hline
\end{tabular}

Fuente: elaboración propia con información de MECASUT (2014). 
El modelo de evaluación MECASUT (2011) clasifica la información en cinco ejes rectores, los cuales están relacionados con los atributos que orientan el quehacer académico de las UT descritos previamente. Los insumos elegidos para el análisis están referidos a cuatro de estos atributos vistos como herramientas desarrolladoras del potencial del estudiante desde la eficiencia, pertinencia, vinculación y equidad, mientras que, por otro lado, los productos se refieren a la eficacia (tabla 2). ${ }^{5} \mathrm{~A}$ continuación, se describen estos atributos a la luz de los indicadores seleccionados.

La eficiencia tiene que ver con la optimización de los recursos para lograr las metas previstas, y en este sentido se emplea la variable tamaño de planta docente. La pertinencia se puede entender como la coherencia entre los programas educativos y las necesidades del entorno, a través, por ejemplo, de proyectos de desarrollo local, regional o nacional; la participación en cuerpos académicos es un indicador del nivel de este atributo.

Las acciones estratégicas orientadas a desarrollar y consolidar la relación con los sectores social y productivo conforman la vinculación, la cual se mide a través del desempeño de bolsa de trabajo. Finalmente, el otorgamiento de becas evalúa la equidad, entendida como el atributo que permite que cualquier grupo social pueda ingresar, permanecer y egresar de la educación superior, con cobertura y calidad (MECASUT, 2011). Si bien no todos los indicadores citados corresponden a insumos en un sentido convencional, sí constituyen capacidades que, en principio, favorecen un mejor desempeño de las instituciones educativas.

Por otro lado, los resultados se refieren a indicadores relacionados en su mayoría con la percepción externa de la calidad del desempeño de la institución en materia de formación (satisfacción de egresados y continuidad de estudios) y la empleabilidad (afinidad/permanencia en empleo y satisfacción de empleadores) (tabla 2). Lejos de verse como un inconveniente, la percepción externa presenta propiedades deseables en términos de imparcialidad. Como se ha dicho, estos indicadores de salida se asocian con la eficacia, es decir, con el nivel de logro de los objetivos y metas programadas para un periodo predeterminado, dados unos recursos.

Aunque no se puede descartar que factores del entorno tales como el sistema económico prevalente (y en concreto el estado del mercado laboral), el nivel de desarrollo social y las políticas públicas tengan influencia en estos indicadores, se asume que son las decisiones y asignaciones institucionales las que producen los mayores efectos en los niveles de estas variables, y que los diferenciales provocados por el entorno están recogidos en las agrupaciones regionales propuestas.

Debido a que la lista de indicadores utilizados no es exhaustiva sino selectiva, ${ }^{6}$ no es posible asegurar causalidad de los insumos hacia los productos, sino más bien, cierta asociación de corto plazo, y por lo tanto, se debe ser cauteloso con el alcance de los indicadores de eficiencia. Lo anterior no va en demérito de la pertinencia de cuestionarse hasta qué punto estos indicadores de salida muestran sensibilidad a las variaciones de los indicadores de entrada.

Cabe destacar que la comparación de los valores medios de los indicadores es muy reveladora. Empezando por los insumos, en términos de tamaño de planta docente, la región sur muestra el valor más alto (40 alumnos por docente) mientras que el norte presenta el valor más bajo (55 alumnos por docente). De la misma manera en materia de otorgamiento de becas, el sur exhibe el valor más alto $(59.4 \%)$ y el norte, el

\footnotetext{
${ }^{5}$ A priori podría parecer contraintuitivo que las variables de salida pertenezcan al eje de eficacia y no al de eficiencia, no obstante, lo que se pretende es que éstas permitan identificar el logro de objetivos y no necesariamente su relación con los insumos, que es justamente el propósito del ejercicio estadístico.

${ }^{6}$ Con base en resultados de la literatura y en los objetivos de investigación.
} 
Tabla 2. Descripción y características de los indicadores de entrada y salida

\begin{tabular}{|c|c|c|c|c|}
\hline Tipo & Eje rector & Clave & Nombre corto & Forma de obtención \\
\hline \multirow{4}{*}{ Entradas } & Eficiencia & E1 & Tamaño de planta docente & Docentes / Alumnos. \\
\hline & Pertinencia & E2 & Participación en cuerpos académicos & Participantes en cuerpos académicos / Docentes de tiempo completo. \\
\hline & Vinculación & E3 & Desempeño de bolsa de trabajo & Egresados colocados por la UT / Egresados. \\
\hline & Equidad & E4 & Otorgamiento de becas & Alumnos becados / Alumnos. \\
\hline \multirow{4}{*}{ Salidas } & Eficacia & S1 & Afinidad/permanencia en empleo & Egresados trabajando en área afín a 6 meses de egreso / Egresados. \\
\hline & Eficacia & S2 & Satisfacción de egresados & Promedio de satisfacción de egresados, base 10. \\
\hline & Eficacia & S3 & Continuidad de estudios & Egresados TSU que siguieron estudiando / Egresados TSU. \\
\hline & Eficacia & $\$ 4$ & Satisfacción de empleadores & Promedio de satisfacción de empleadores, base 10. \\
\hline
\end{tabular}

Fuente: elaboración propia con información de MECASUT (2011).

más bajo (39.1\%); lo cual se puede interpretar como resultado de la implementación de medidas en busca de la equidad social. En el caso de la participación en cuerpos académicos y desempeño de bolsa de trabajo el norte presenta los indicadores más elevados $(47.2 \%$ y $31.2 \%$, respectivamente).

En lo que respecta a los productos, el norte muestra los valores más elevados en términos de afinidad/permanencia en empleo (38.1\%) y en satisfacción de egresados (8.7 de calificación), que puede ser una manifestación de mayores y mejores oportunidades laborales, asociadas, en parte, al nivel de los insumos. Por otra parte, los valores más altos en continuidad de estudios (89.6\%) y en satisfacción de empleadores (8.9 de calificación) los presenta la región sur. Lo anterior evidencia una inconsistencia entre la satisfacción de los egresados y la de los empleadores, que ni siquiera la lógica de los flujos migratorios puede alcanzar a explicar y que puede tener que ver con la existencia de expectativas diferenciadas entre los empleadores de las distintas regiones, según las actividades económicas preponderantes.
En las figuras 2, 3, 4 y 5 se representa el problema que implica la minimización de una entrada y la maximización de una salida, a través de gráficos de dispersión de los indicadores incluidos en el análisis. Es importante destacar que el ejercicio tiene la intención de visualizar el grado de correlación entre pares de indicadores y, al mismo tiempo, identificar patrones de comportamiento por región, por lo que no se utiliza de forma simultánea la información de todas las variables, pero sí la de todas las UT bajo análisis. Las mejores relaciones salida/entrada se unen con una línea punteada que forma una hipotética frontera eficiente suponiendo rendimientos variables a escala. En cada gráfico se agrega una línea de tendencia que representa la relación lineal entre las variables.

La figura 2 muestra la relación entre el tamaño de planta docente y cada una de las salidas. Aunque Lucena y Moreira (2015) lo consideran un indicador relevante en la evaluación de la eficiencia, en este trabajo resultó ser la entrada con mayor ausencia de correlación positiva con las salidas consideradas. También se trata del caso donde, en promedio, un 
mayor número de UT conforman la frontera eficiente, en la cual están presentes mayoritariamente las regiones norte y centro-este. Se destaca la poca dispersión relativa de las universidades de la región centro-oeste en términos de satisfacción de egresados, lo que habla de una mayor estandarización de los resultados.

La figura 3 presenta la relación entre la participación en cuerpos académicos y cada una de las salidas. Se encontraron tres correlaciones ligeramente positivas, pero también una negativa: específicamente aquella con la satisfacción de empleadores. Mazeran et al. (2006) ya identificaban un escaso grado de adecuación entre los esfuerzos de investigación y las carencias del aparato productivo. Si bien no se puede descartar que estos esfuerzos tengan un efecto favorable en la calidad de los egresados, los mismos no parecen ser identificados adecuadamente por los empleadores. Las regiones que con mayor frecuencia conforman una frontera hipotética son nuevamente la norte y la centro-este, con alguna aparición esporádica de la zona centro-oeste.

Figura 2. Gráfico de dispersión entre las salidas y la entrada "tamaño de planta docente"
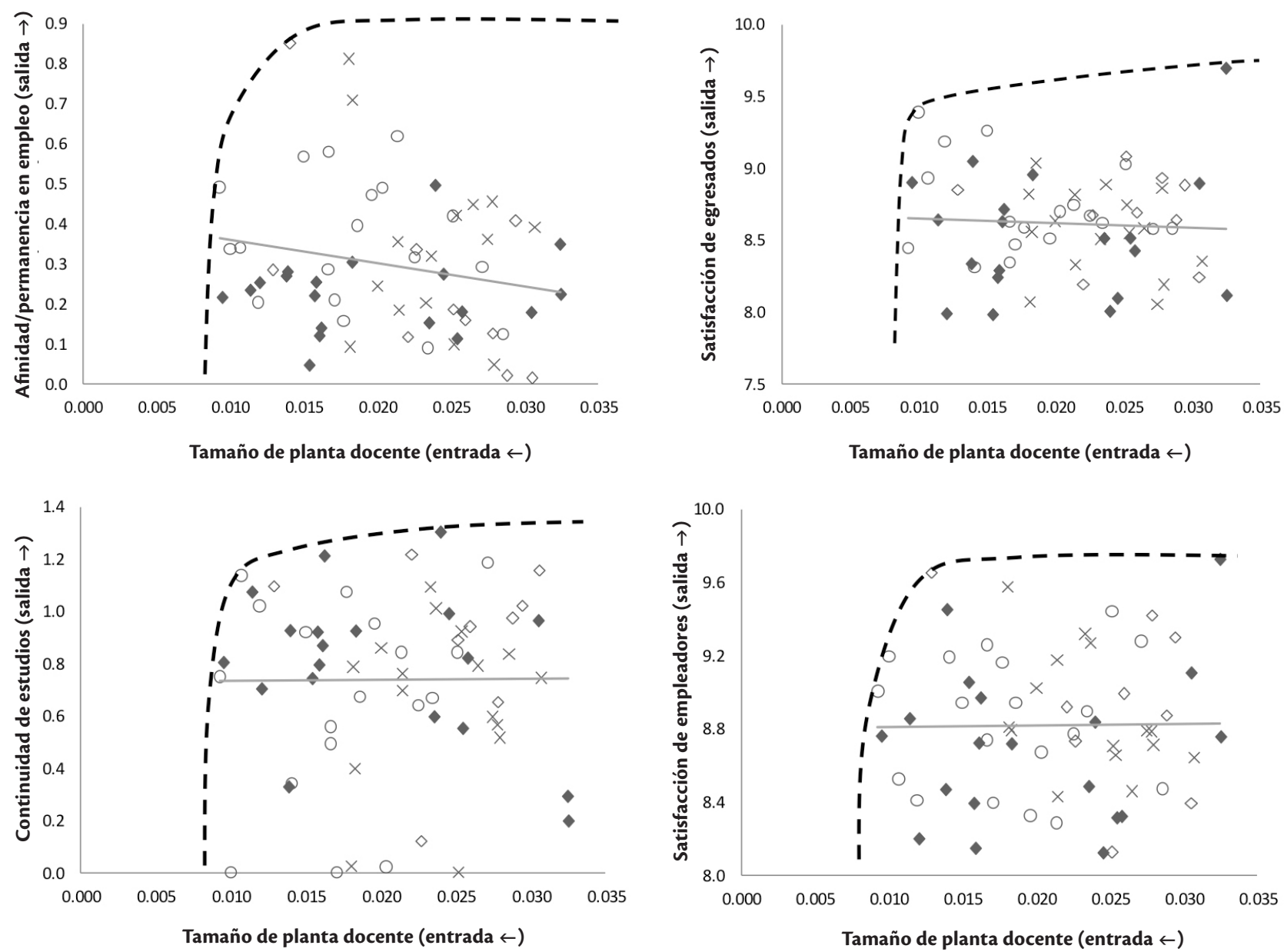

Fuente: elaboración propia con información de MECASUT (2014). O: UT región norte. $\diamond:$ UT región sur. X· UT región centro-oeste. UT región centro-este.

${ }^{7}$ Este hecho se repite en la mayoría de las figuras debido a que se mantienen las mismas variables en el eje de ordenadas. 

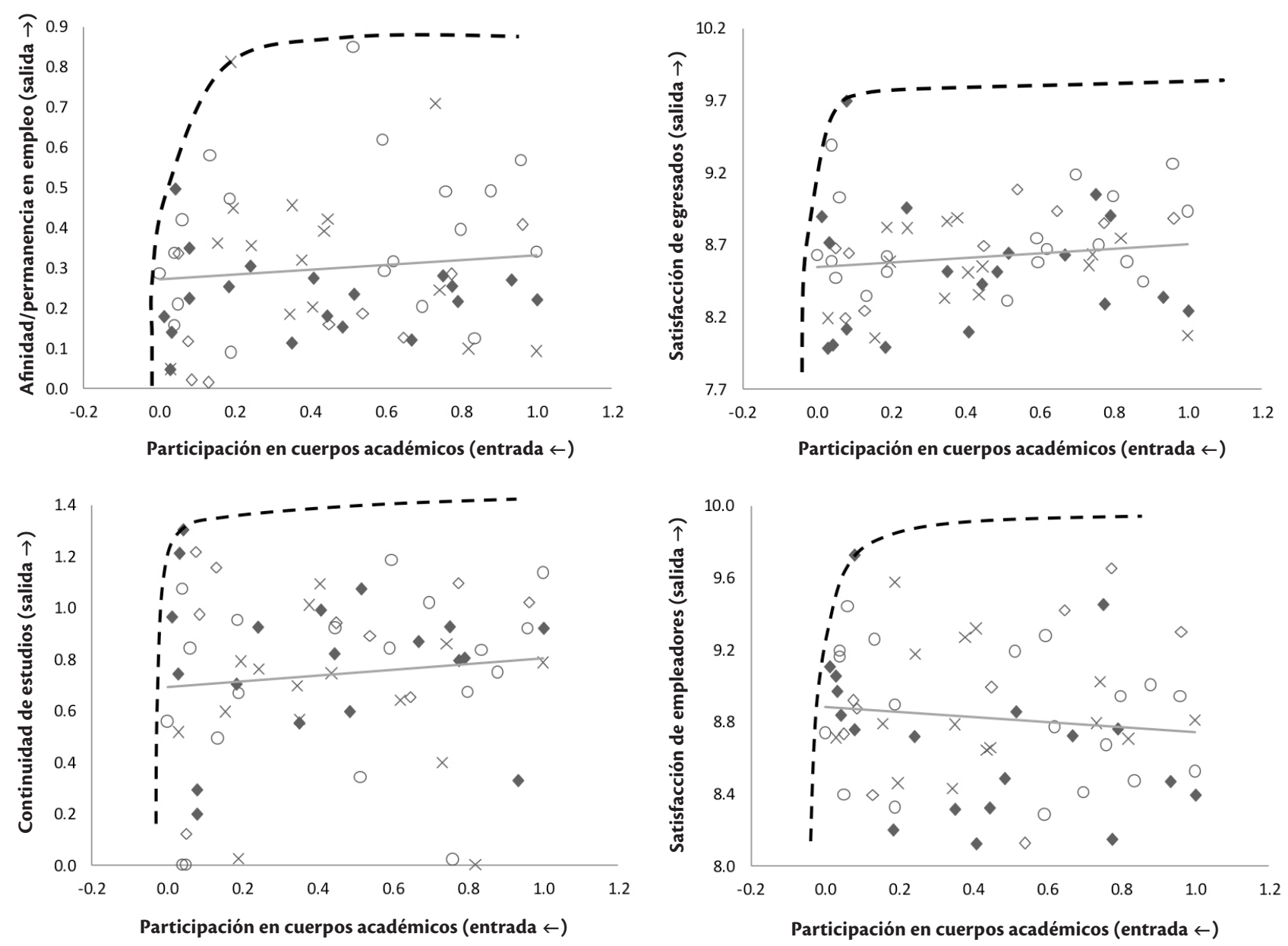

Fuente: elaboración propia con información de MECASUT (2014). O: UT región norte. $\diamond:$ UT región sur. X: UT región centro-oeste. UT región centro-este.

La asociación entre los resultados y el desempeño de bolsa de trabajo se muestra en la figura 4. Se presentan tres correlaciones positivas y una negativa, relacionada con la continuidad de estudios. Este hallazgo es hasta cierto punto justificable pues la colocación laboral puede hacer que la opción de seguir estudiando sea pospuesta. Dada la poca dispersión de los valores en el indicador de insumo, se debe tomar con reserva la propuesta de frontera eficiente. Con relación a lo anterior, es la región norte la que presenta la mayoría de los casos con valores significativamente distintos de cero en el desempeño de bolsa de trabajo.

La figura 5 presenta la asociación entre el otorgamiento de becas y cada una de las salidas. Se presentan dos correlaciones positivas, una negativa (con la afinidad/permanencia en empleo para la cual nuevamente se podría encontrar justificación en los incentivos de los agentes) y una falta de relación. Al igual que en el resto de figuras, las regiones que suelen conformar la frontera son la norte y centro-este. 
Figura 4. Gráfico de dispersión entre las salidas y la entrada “desempeño de bolsa de trabajo"
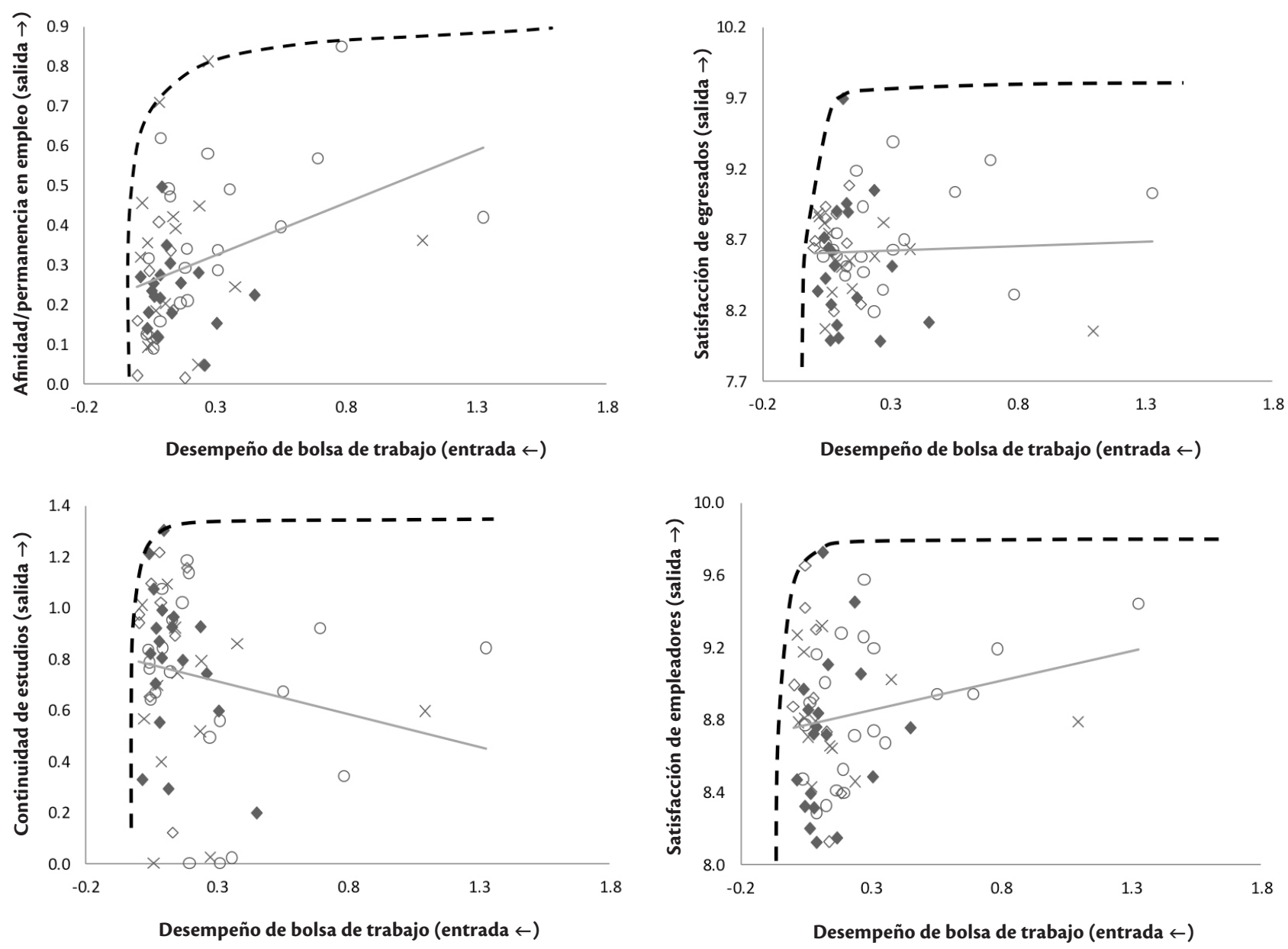

Fuente: elaboración propia con información de MECASUT (2014). O: UT región norte. $\diamond$ : UT región sur. X: uT región centro-oeste. UT región centro-este.

Continuando con la figura 5, la región centro-este es la que presenta una mayor proporción de sus UT por debajo de la línea de tendencia en los casos de afinidad/permanencia en empleo y satisfacción de empleador — seguida de cerca por la región sur-, apareciendo por encima de esta línea cuando se trata de la continuidad de estudios, ${ }^{7}$ lo que denota mayor inestabilidad laboral, pero al mismo tiempo mejor aprovechamiento de los apoyos para continuar los estudios en estas regiones. 
Figura 5. Gráfico de dispersión entre las salidas y la entrada "otorgamiento de becas"
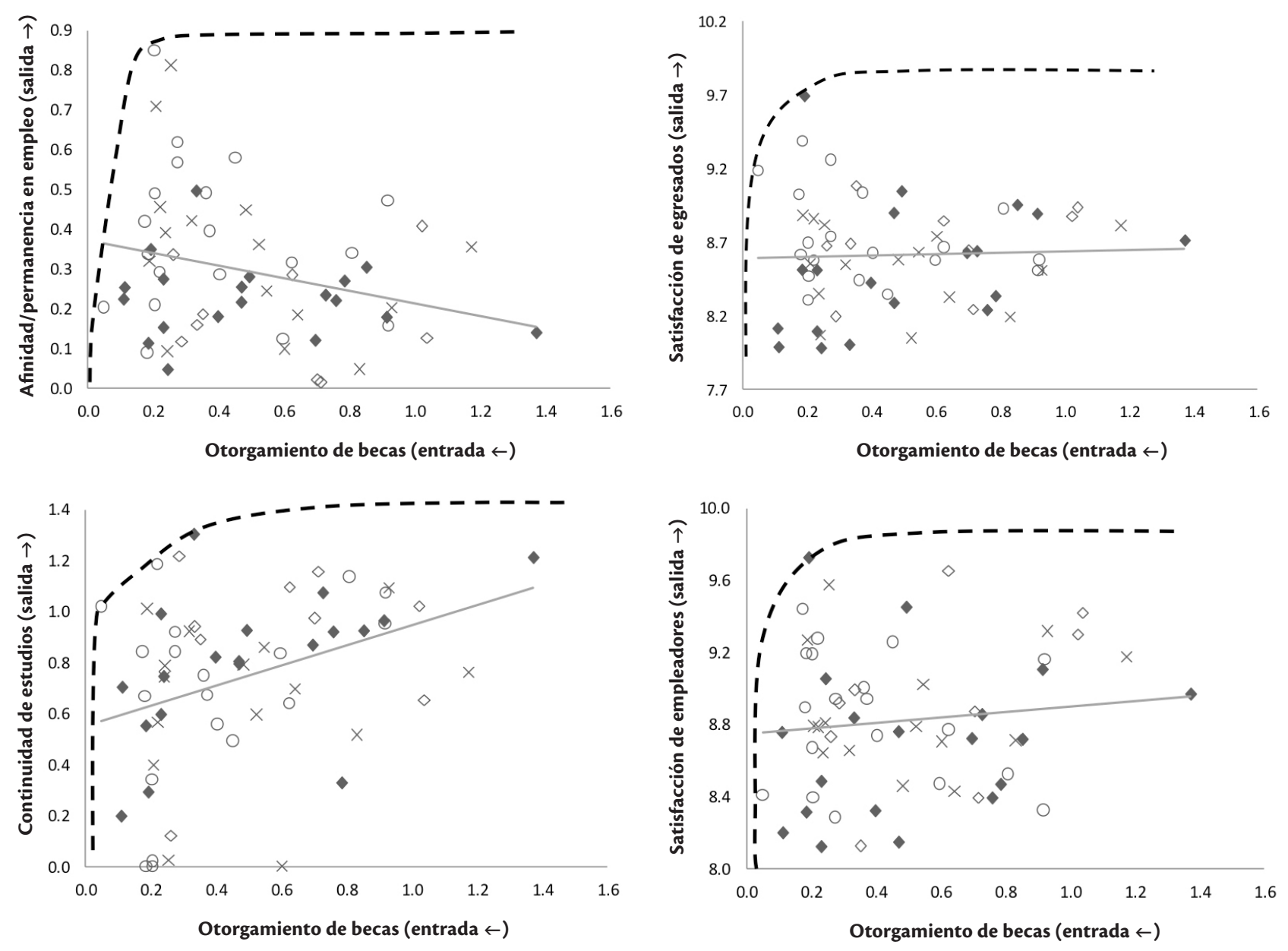

Fuente: elaboración propia con información de MECASUT (2014). O: UT región norte. $\diamond:$ UT región sur. X: UT región centro-oeste. UT región centro-este.

\section{Procedimiento de análisis de datos}

De acuerdo con la teoría económica, la eficiencia consiste en asegurar el correcto uso de los medios empleados para alcanzar ciertos fines (Quindós et al., 2003); su medición se basa en comparar la actuación real de una organización con respecto a un óptimo. A nivel global, existe una preocupación creciente con relación a la medición y comparación de la eficiencia en instituciones tales como escuelas, hospitales, empresas, bancos, gobiernos y otras donde se dispone de un conjunto de capacidades o recursos
- tecnológicamente comparables y en ese sentido, relativamente homogéneos - para conseguir unos objetivos. Para Nazarko y Saparauskas (2014), la educación superior pública en todo el orbe también está sujeta a una creciente presión por incrementar su calidad, eficiencia e impacto.

Retomando la discusión acerca de la medición de la eficiencia, lo ideal sería comparar lo que hace la institución con lo que podría haber hecho para alcanzar sus objetivos; sin embargo, no se suele tener información completa acerca del contexto en el que 
se desenvuelve cada organización, ni de la tecnología o las restricciones que actúan sobre la misma, la cual pueden afectar el nivel de logro posible (Álvarez, 2001). Por lo tanto, lo mejor que se puede hacer es comparar la actuación de la organización con el de otras parecidas, bajo el supuesto de que los objetivos de todas son similares.

Con miras a la determinación de la eficiencia relativa, Charnes et al. (1978) reconocen la dificultad de encontrar un conjunto de pesos en las variables de un problema, debido a que las unidades de decisión pudieran valorar de forma diferente - pero en todo caso, legítima-, los insumos y productos. Debido a lo anterior, los autores propusieron que a cada unidad se le permita adoptar el conjunto de pesos que pueda mostrarla de la forma más competitiva en el entorno de una hipotética frontera eficiente, definida por la actuación de las mejores organizaciones. El enfoque no paramétrico para el cálculo empírico de esta frontera admite la construcción de supuestos sobre las propiedades de la tecnología de producción que, a su vez, permiten definir, con el apoyo de los datos observados, el conjunto de planes de producción realizables. Así, esta aproximación no requiere que se asuma una forma funcional para la frontera, tal como ocurre con las aproximaciones paramétrica.

$\mathrm{El}$ análisis envolvente de datos (AED) es una técnica de programación lineal que permite estudiar la eficiencia de una organización en su contexto productivo con relación al comportamiento de otras similares, a partir de la construcción de una frontera eficiente mediante aproximaciones no paramétricas. El modelo AED versión BCC (Banker et al., 1984) está basado en rendimientos variables a escala, midiendo exclusivamente ineficiencias debidas a la gestión productiva. Por otro lado, la versión CCR (Charnes et al., 1978) permite identificar diferencias en las escalas productivas de las unidades sujetas a medición. Este último fue el modelo originalmente propuesto para la estimación de la eficiencia en contextos tradicionales.
En términos generales, el AED sugiere que, dado un proceso productivo en el que se utilizan $p$ entradas $\left(x_{p}, x_{2}, \ldots x_{p}\right)$ en la producción de $q$ salidas $\left(y_{1}, y_{2}, \ldots y_{p}\right)$ y para el que se cuenta con observaciones correspondientes a $n$ unidades productivas, la eficiencia técnica de una determinada unidad 0 puede estimarse a través del siguiente programa lineal:

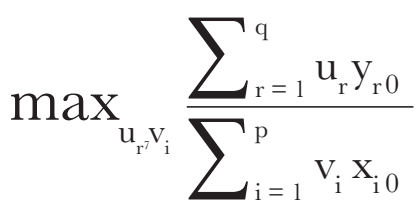

sujeto a

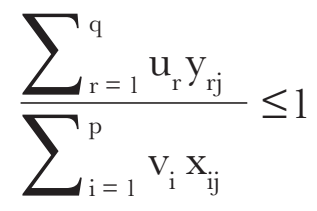

donde $j=1, \ldots, n, r=1, \ldots, q$ y $i=1, \ldots, p$. Además $u, v, \geq e$, donde $e$ representa un valor infinitesimalmente pequeño pero mayor que cero.

La idea subyacente es la de maximizar un índice de productividad total de los factores (por unidad de insumo empleada) para cada unidad de decisión. Sin embargo, para esta agregación se utiliza un conjunto de ponderaciones $\left(u_{p}, \ldots u_{q}, v_{p}, \ldots v_{p}\right)$, cuyo valor es precisamente el que se pretende hallar, de tal forma que se maximice el ratio para cada unidad de decisión y al mismo tiempo se consiga que los ratios del resto sean menores que uno. En la búsqueda de una solución, es conveniente linealizar el algoritmo presentado para poder aplicar los métodos de programación lineal. A través de la resolución del problema fraccional original o de cualquiera de sus formas lineales, se obtiene la eficiencia estimada de la unidad 0 , que tomará el valor 1 si ésta es técnicamente eficiente. Un problema similar debe ser resuelto simultáneamente para las $n$ - 1 unidades restantes.

El AED constituye una herramienta objetiva porque no precisa de la asignación de ponderaciones $a$ 
priori (lo cual también podría constituir un problema en el caso de ponderaciones nulas), y es considerablemente flexible porque no exige que las unidades de decisión concedan la misma importancia a los indicadores (Martínez et al., 2005). Por lo tanto, el AED puede ser apropiado cuando las unidades sujetas a medición pueden valorar - justificadamente - entradas o salidas de manera diferente, o donde hay alta incertidumbre acerca de sus valores. Además, permite graduar la flexibilidad a través de restricciones adicionales que reflejen las relaciones conocidas entre las entradas y las salidas. Por último, Johnes (2006) afirma que el AED es una opción atractiva para medir la eficiencia de las instituciones de educación superior pero también advierte que sus inconvenientes no deben ser pasados por alto. ${ }^{8}$

\section{Resultados}

Hay algunos supuestos y aspectos operacionales que conviene destacar antes de presentar los resultados. Primero, se supondrá que el nivel de las salidas es sensible al de las entradas a nivel de cada unidad de decisión (las sedes del Sistema de Universidades Tecnológicas) y que las decisiones de estas unidades tienen impacto sobre sus niveles. En segundo lugar, aunque todos los indicadores incluidos son del tipo "entre más, mejor", debe tenerse presente que las erogaciones destinadas a incrementar el nivel de los insumos tienen asociados costos de oportunidad que podrían afectar la conformación del presupuesto institucional.

Finalmente, en la estimación de la eficiencia ${ }^{9}$ se utiliza una orientación a las entradas, es decir, la frontera se calcula minimizando los insumos dado el nivel de productos. La naturaleza de los insumos seleccionados hace suponer que los planteles tienen influencia en sus niveles, a diferencia de otros trabajos que dan más peso a la flexibilidad los productos mientras que consideran los insumos como de naturaleza rígida (Nazarko y Saparauskas, 2014; Ghose, 2017). Una vez hechas estas precisiones se procede a la descripción de los hallazgos.

Las tablas 3, 4, 5 y 6, presentan los niveles de eficiencia técnica global (ETG), eficiencia técnica pura (ETP) y eficiencia de escala (EE) para cada región (Goñi, 1998), ${ }^{10}$ en cuyo cálculo se requirió del AED tanto versión BCG como CGR (Quindós et al., 2003). Además de este resultado básico, se proporciona información adicional, por ejemplo: el conjunto de referencia para las unidades ineficientes (presentadas por orden de importancia), los niveles de holgura, entendidos como los ajustes necesarios para que la unidad aspire a la eficiencia y la aportación de cada indicador al índice de referencia estimado. Tanto las holguras (tabla 7) como las UT de referencia (última columna en tablas 3, 4, 5 y 6) están referidas al modelo de retornos variables de escala, es decir, no toman en cuenta las ineficiencias atribuidas exclusivamente a la escala. ${ }^{11}$

Los promedios de ETG, ETP y EE en las UT de la región norte (tabla 3), fueron respectivamente, $96.7 \%, 97.5 \%$ y $99.1 \%$, mientras que el porcentaje de UT que trabajó en la frontera eficiente fue $84.2 \%$, $89.5 \%$ y $84.2 \%$. Las UT ineficientes muestran, en todos los casos, una dimensión pequeña respecto al óptimo (retornos decrecientes). Las principales universidades con las que se comparan los casos ineficientes de este grupo son Chihuahua y Matamoros, sobre todo, por su aprovechamiento en materia de tamaño de planta docente y otorgamiento de becas.

\footnotetext{
${ }^{8}$ Por ejemplo, podría darse una incorrecta definición de entradas y salidas, falta de homogeneidad de las unidades de análisis o falta de fiabilidad de los datos.

${ }^{9}$ Con las reservas descritas en la sección de metodología y datos.

${ }^{10}$ La eficiencia técnica global depende de la ocurrencia de la eficiencia técnica pura (aspectos operacionales) y la eficiencia de escala (aspectos dimensionales). La eficiencia de escala resulta del cociente entre la eficiencia técnica global y la eficiencia técnica pura.

${ }^{11}$ Cabe señalar que, al tratarse de ejercicios independientes por región, la comparación de la eficiencia individual de UT de diferentes regiones carece de sentido estadístico.
} 
En la región sur, los promedios de ETG, ETP y EE fueron $92.1 \%, 96.9 \%$ y $95.2 \%$, respectivamente, y el porcentaje de UT trabajando en la frontera, $55.6 \%$, $88.9 \%$ y $55.6 \%$ (tabla 4 ). Nuevamente, las ineficiencias se relacionan con un retorno decreciente de los factores productivos. El análisis sugiere que la UT de Valles-Centrales-Oaxaca podría tomar como referencia el desempeño de la UT Región-Norte-Guerrero que ha hecho un mejor trabajo en lo que se refiere a la participación en cuerpos académicos y otorgamiento de becas.

Los datos medios de eficiencia de la región centrooeste (tabla 5), indican que $73.3 \%, 80.0 \%$ y $73.3 \%$ de las UT trabajaron correspondientemente en la frontera de ETG, ETP y EE, mientras que 96.7\%, $97.7 \%$ y $98.9 \%$ fueron los promedios de eficiencia observados en la región. Presentan una dimensión insuficiente las UT Suroeste-Guanajuato y NorteAguascalientes, mientras que Norte-Guanajuato y Jalisco muestran un producto superior al esperado. ${ }^{12}$

Curiosamente, ésta es la región más asimétrica en cuanto a los retornos de escala, sin embargo, no hay casos con eficiencias muy bajas lo que indica una moderada discriminación general. Las universidades de mejores prácticas con las que se comparan los casos ineficientes de este grupo son Nayarit y Bahía-Banderas. La primera ha sido más eficiente regionalmente con relación al tamaño de planta docente y el desempeño de bolsa de trabajo; en el caso de la segunda UT hay que añadir a los anteriores indicadores, el otorgamiento de becas.

Tabla 3. Resultados: casos con algún tipo de ineficiencia en región norte

\begin{tabular}{|c|c|c|c|c|c|}
\hline UT & ETG & ETP & EE & Retornos & Referencias \\
\hline Coahuila & 80.0 & 84.1 & 95.2 & Decreciente & Chihuahua \\
\hline Torreón & 65.3 & 68.2 & 95.7 & Decreciente & Matamoros \\
\hline
\end{tabular}

Fuente: elaboración propia. ETC: eficiencia técnica global. ETP: eficiencia técnica pura. EE: eficiencia escala. Las UT Hermosillo, Nogales, Tijuana, Ciudad-Juárez, Chihuahua, Sur-Sonora, Durango, Izúcar-Matamoros, Gral-Mariano-Escobedo, Norte-Coahuila, Santa-Catarina, Tamaulipas-Norte, Matamoros, Altamira, Nuevo-Laredo, Cadereyta resultaron eficientes y presentaron retornos constantes.

Tabla 4. Resultados: casos con algún tipo de ineficiencia en región sur

\begin{tabular}{|c|c|c|c|c|c|}
\hline UT & ETG & ETP & EE & Retornos & Referencias \\
\hline $\begin{array}{c}\text { Valles-Centrales- } \\
\text { Oax. }\end{array}$ & 71.6 & 72.0 & 99.4 & Decreciente & Región-Norte-Gro \\
\hline Tabasco & 72.5 & 100.0 & 72.5 & Decreciente & Región-Norte-Gro \\
\hline Campeche & 92.0 & 100.0 & 92.0 & Decreciente & Región-Norte-Gro \\
\hline Metropolitana & 92.9 & 100.0 & 92.9 & Decreciente & Región-Norte-Gro \\
\hline
\end{tabular}

Fuente: elaboración propia. ETC: eficiencia técnica global. ETP: eficiencia técnica pura. EE: eficiencia escala. Las UT Costa-Grande-Gro., Selva, RegiónNorte-Gro., Cancún, San-Juan-del-Río resultaron eficientes y presentaron retornos constantes.

\footnotetext{
${ }^{12}$ Es probable que este tipo de ineficiencia sea el resultado de inversiones o cambios estructurales no reflejados en los insumos seleccionados.
} 
En la región centro-este, $57.9 \%, 73.7 \%$ y $57.9 \%$ de las UT trabajó en la frontera de ETG, ETP y EE (tabla 6) y el promedio de eficiencia observado de ETG fue $90.8 \%, 94.7 \%$ y $95.6 \%$, respectivamente. Presentaron niveles inferiores a los esperados de acuerdo con las referencias regionales, Tecámac, Tula-Tepeji, Puebla, Huasteca-Hidalguense, Tlaxcala, Valle-Mezquital y Centro-Veracruz, mientas que Tecamachalco exhibió resultados superiores a los esperados de acuerdo con el nivel de sus factores. Para ser eficientes las mencionadas necesitarían transitar hacia las prácticas presentadas por la UT "Emiliano Zapata"-Morelos, que ha sido eficiente con relación al tamaño de planta docente y el desempeño de bolsa de trabajo, y la UT Fidel-Velázquez, que además de las anteriores, también presentó buenos resultados en otorgamiento de becas.

A continuación, se presentan los casos de UT débilmente eficientes en el sentido de que al menos una entrada o salida presenta una holgura distinta de 0 debido a problemas con la escala. Una holgura en las salidas representa el nivel adicional de éstas, requerido para conseguir la eficiencia de referencia; por su parte, una holgura en las entradas establece las reducciones necesarias en éstas para alcanzar el objetivo planteado.

Tabla 5. Resultados: casos con algún tipo de ineficiencia en región centro-oeste

\begin{tabular}{|c|c|c|c|c|c|}
\hline UT & ETG & ETP & EE & Retornos & Referencias \\
\hline Norte-Guanajuato & 94.5 & 98.9 & 95.6 & Creciente & Nayarit \\
\hline Suroeste-Guanajuato & 81.6 & 83.7 & 97.5 & Decreciente & Bahía-Banderas \\
\hline Norte-Aguascalientes & 94.8 & 100.0 & 94.8 & Decreciente & Bahía-Banderas \\
\hline Jalisco & 79.2 & 83.0 & 95.4 & Creciente & Bahía-Banderas \\
\hline
\end{tabular}

Fuente: elaboración propia. ETG: eficiencia técnica global. ETP: eficiencia técnica pura. EE: eficiencia escala. Las UT Aguascalientes, Querétaro, León, San-LuisPotosi, Zacatecas, Salamanca, Morelia, Nayarit, Costa-Nayarit, Zona-Metrop.-Gdl., Bahía-Banderas resultaron eficientes y presentaron retornos constantes.

Tabla 6. Resultados: casos con algún tipo de ineficiencia en región centro-este

\begin{tabular}{|c|c|c|c|c|c|}
\hline UT & ETG & ETP & EE & Retornos & Referencias \\
\hline Tecámac & 90.0 & 100.0 & 90.0 & Decreciente & \\
\hline Tula-Tepeji & 54.0 & 72.1 & 75.0 & Decreciente & Fidel-Velázquez \\
\hline Puebla & 81.3 & 93.6 & 86.9 & Decreciente & Fidel-Velázquez \\
\hline Huasteca-Hidalguense & 82.9 & 100.0 & 82.9 & Decreciente & \\
\hline Tecamachalco & 78.2 & 79.3 & 98.6 & Creciente & "E.-Zap."-Mor. \\
\hline Tlaxcala & 73.1 & 73.4 & 99.6 & Decreciente & Tula-Tepeji \\
\hline Valle-Mezquital & 74.5 & 80.3 & 92.7 & Decreciente & “E.-Zap."-Mor. \\
\hline Centro-Veracruz & 91.3 & 100.0 & 91.3 & Decreciente & \\
\hline
\end{tabular}

Fuente: elaboración propia. ETC: eficiencia técnica global. eTP: eficiencia técnica pura. EE: eficiencia escala. Las UT Nezahualcóyotl, Fidel-Velázquez, Sur-Edo. México, "E.-Zap."-Mor., Valle-Toluca, Tulancingo, Sierra-Hidalguense, Huejotzingo, Xicotepec-Juárez, Sureste-Veracruz, Gutiérrez-Zamora resultaron eficientes y presentaron retornos constantes. 
Tabla 7. Resultados de holguras para la región centro-este

\begin{tabular}{|c|c|c|c|c|c|c|c|c|c|}
\hline \multirow{2}{*}{ Región } & \multirow{2}{*}{ UT } & \multicolumn{4}{|c|}{ Holguras de entrada } & \multicolumn{4}{|c|}{ Holguras de salida } \\
\hline & & E1 & E2 & E3 & E4 & s1 & s2 & s3 & 54 \\
\hline \multirow{2}{*}{ Norte } & Coahuila & - & 2.7 & - & - & 7.4 & 80.3 & - & 90.0 \\
\hline & Torreón & - & 0.2 & - & - & 75.0 & 54.0 & 72.1 & 75.0 \\
\hline Sur & $\begin{array}{c}\text { Valles-Centrales- } \\
\text { Oaxaca }\end{array}$ & - & 5.4 & 21.0 & 11.6 & - & 53.5 & 93.6 & 86.9 \\
\hline \multirow{3}{*}{ Centro-oeste } & Norte-Guanajuato & - & 24.7 & 46.0 & - & 79.0 & 82.9 & 100.0 & 82.9 \\
\hline & Suroeste-Guanajuato & - & 22.3 & - & 35.7 & - & 72.6 & 37.0 & 98.6 \\
\hline & Jalisco & 0.3 & - & 7.4 & - & - & 51.7 & 12.6 & 65.4 \\
\hline \multirow{5}{*}{ Centro-este } & Tula-Tepeji & - & 13.5 & 14.3 & - & 12.1 & - & 1.2 & 15.8 \\
\hline & Puebla & 0.4 & 13.8 & - & 15.7 & - & 5.5 & 31.9 & 86.9 \\
\hline & Tecamachalco & - & 30.3 & - & 1.8 & 24.0 & - & 27.7 & 82.9 \\
\hline & Tlaxcala & - & 3.8 & 4.3 & - & - & 24.4 & 2.6 & 44.7 \\
\hline & Valle-Mezquital & - & 11.8 & - & 1.7 & - & 51.7 & 12.6 & 65.4 \\
\hline
\end{tabular}

Fuente: elaboración propia. E1: tamaño de planta docente. E2: participación en cuerpos académicos. E3: desempeño de bolsa de trabajo. E4: otorgamiento de becas. S1: afinidad/permanencia en empleo. 52: satisfacción de egresados. 53: continuidad de estudios. 54: satisfacción de empleadores.

De la tabla 7 se destaca la necesidad, para la región norte, de incrementar hasta en $80 \%$ el nivel de continuidad de estudios en una de sus UT. Llama la atención, asimismo, el caso de la región centro-este, donde cuatro de las cinco universidades con holguras, mostraron exceso en el nivel de participación en cuerpos académicos dados los resultados regionales; al mismo tiempo todas las UT de esta región, junto con las del sur y centro-oeste, presentaron un déficit en la satisfacción de empleadores.

La contribución relativa de cada indicador refleja los puntos fuertes y débiles en el funcionamiento y operación de las unidades de medición, de tal forma que, de presentarse una ventaja relativa en un indicador (ya sea de entrada o de salida) lo racional es que la unidad busque obtener de éste una mayor contribución (Martínez y Murias, 2011). Así, en materia de comportamientos medios en la gestión de insumos, las cuatro regiones dieron un mayor peso al tamaño de planta docente, debido quizá a que éste es el insumo en el que la institución puede incidir de forma más expedita, aunque, por otro lado, se encontró que, a nivel individual, este indicador no parece tener demasiada indecencia en los resultados. No obstante, el comportamiento fue diferenciado en lo que se refiere al insumo con menos peso; siendo éste, otorgamiento de becas para la región norte, desempeño de bolsa de trabajo para el centro-oeste y sur, y participación en cuerpos académicos para el centro-este.

En lo que se refiere a la gestión de resultados, todas las regiones dieron un mayor peso a satisfacción de egresados, exceptuando la centro-oeste que lo hizo a la continuidad de estudios. También todas, excluyendo el centro-oeste, dieron una menor ponderación a la satisfacción de empleadores, mientras que esta última, lo hizo a la satisfacción de egresados. 


\section{Conclusiones}

A través del análisis envolvente de datos, en este trabajo se lleva a cabo una aproximación al análisis de la eficiencia técnica regional de las universidades tecnológicas de México en lo que se refiere al potencial para impactar el mercado de trabajo, las expectativas laborales y la formación continua de sus egresados, a través de la medición del aprovechamiento de ciertos insumos para la consecución de un conjunto de objetivos.

Los estadísticos básicos de los indicadores de insumo mostraron que la región sur tiene los valores medios más altos en cuanto al apoyo a los estudiantes desde un punto de vista de la equidad social, mientras que el norte exhibe los valores más bajos; no obstante, en materia de investigación y colocación laboral ocurrió justo lo inverso. En lo que respecta a los indicadores de salida, el norte muestra los valores más elevados en cuanto a afinidad y permanencia laboral, así como en la satisfacción de los egresados, mientras que los promedios más altos en términos de los esfuerzos por continuar los estudios y la satisfacción de los empleadores, los presenta la región sur. El hallazgo de esta polarización no es sorprendente, sí llama la atención que ninguna de estas regiones tenga en exclusividad los peores niveles de los indicadores, lo que habla de la presencia de desequilibrios entre regiones, flujos migratorios y visiones diferenciadas del concepto de desarrollo.

Considerando que las fronteras de eficiencia técnica global de las regiones son distintas entre sí, se puede afirmar que es en la región norte donde un mayor porcentaje de universidades trabaja sobre esta frontera, mientras que en la región sur es donde lo hacen una menor proporción de universidades, seguida muy de cerca por la región centro-este. Cabe destacar que también son estas dos últimas regiones las que presentan los peores promedios de eficiencia.
Por otra parte, los promedios regionales de eficiencia técnica global y de escala fueron también más altos en la región norte; sin embargo, la región con la mayor eficiencia técnica pura fue la centro-oeste. Lo anterior es evidencia de que, en la búsqueda de sus objetivos, las universidades tecnológicas no tienen los mismos criterios de decisión y oportunidades de aprovechamiento de los insumos, derivado de lo cual existen importantes diferencias en su desempeño.

Aunque la mayoría de las correlaciones entre indicadores de insumo y de producto resultó al menos ligeramente positiva como se esperaba, ${ }^{13}$ se presentaron casos tales como el del tamaño de la planta docente que no mostraron capacidad para incrementar la afinidad/permanencia en empleo, lo que refleja un problema en los mecanismos de asignación de recursos para estas áreas. Haciendo una generalización en cuanto a la gestión de insumos, las cuatro regiones se inclinan por el aprovechamiento de los elementos de eficiencia asociados al manejo de la planta docente antes que por aspectos de vinculación y pertinencia. Por otro lado, la gestión de resultados está soportada preponderantemente en la satisfacción de los egresados, quedando en segundo término los aspectos que van más allá del egreso como lo son la afinidad laboral, la continuidad de los estudios, o incluso, la satisfacción de los empleadores. ${ }^{14}$ Lo anterior pone de manifiesto la necesidad de buscar nuevas fórmulas de asignación y aprovechamiento de recursos para que este sistema de universidades, cuyos fines son sabidamente pertinentes y consistentes con los retos que enfrenta el país, logre jugar el rol que le corresponde en el sistema educativo mexicano.

Aunque el trabajo contribuye al análisis estadístico-empírico del sistema educativo de universidades tecnológicas, esta aportación no estuvo exenta de restricciones. Entre las principales limitaciones del trabajo están las referidas a la falta de asignación de

\footnotetext{
${ }^{13} \mathrm{O}$ en su defecto se encontraron argumentos evidentes para explicar la falta de relación.

${ }^{14}$ Sin que ello signifique que no haya casos concretos que sí basen su eficiencia en el manejo de estos últimos indicadores.
} 
ponderaciones a priori en el modelo y el uso selectivo de indicadores. También se debe mencionar que se trata un análisis parcial, al no haber podido incluirse - por limitaciones técnicas-, todas las variables $a$ priori relevantes, pero sí las que resultan más representativas, de acuerdo con la literatura y los objetivos del estudio.

Por otro lado, las líneas de investigación futura versan sobre la inclusión de criterios diversificados

\section{Referencias}

Álvarez, Antonio (coord.) (2001), La medición de la eficienciay la productividad, Madrid, Ed. Pirámide.

Banker, Rajiv, Abraham Charnes y William Cooper (1984), "Some models for estimating technical and scale efficiencies in data envelopment analysis", Management Science, vol. 30, núm. 9, pp. 1078-1092.

Bradley, Steve, Jill Johnes y Allan Little (2010), "Measurement and determinants of efficiency and productivity in the further education sector", Bulletin of Economic Research, vol. 62, núm. 1, pp. 1-30.

CGUT-SEP (2004), La evaluación externa en las universidades tecnológicas. Un medio eficaz para la rendición de cuentas. Informes y recomendaciones 1996, 1999 y 2002, México, Limusa-THH.

Charnes, Abraham, William Cooper y Eduardo Rhodes (1978), "Measuring the efficiency of decision making units", European Fournal of Operational Research, vol. 2, núm. 6, pp. 429-444.

Flores-Crespo, Pedro (2009), Trayectoria del modelo de universidades tecnológicas en México (1991-2009) [Cuadernos de Trabajo de la Dirección General de Evaluación Institucional (DGEI)], México, UNAM.

Fouquet, Anne (2002), "Diferencias regionales en México: una herencia geográfica y política", en N. Guzmán (comp.), Sociedad y Desarrollo en México, Monterrey, Ediciones Castillo/ITESM, pp. 385-402. por región (variables ambientales) que permitan la comparación nacional y la exploración de otras agrupaciones regionales posibles. Además, la discusión se enriquecería notablemente de poder ser incluidas las líneas de acción oficiales seguidas por las instituciones educativas en materia de empleabilidad, lo cual permitiría cotejar el desempeño de cada plantel no sólo con el de los mejores de su región, sino también con las acciones que él mismo emprendió.

García, Carlos (2009), “Corporación Universitaria para el Desarrollo de Internet”, Reunión de Otoño 2009, México, Sistema Nacional de Educación Superior Tecnológica (SNEST-CUDI), <http://www.cudi.edu. mx/otono_2009/presentaciones/carlos_alonso.pdf> [consulta: septiembre de 2017].

Ghose, Arpita (2017), Efficiency of elementary education in India: empirical evidence using a nonparametric data envelopment approach, India, SpringerBriefs in Economics.

González, Pablo (2001), La universidad necesaria en el siglo XXI, México, Ediciones Era.

Goñi, Salomé (1998), "El análisis envolvente de datos como sistema de evaluación de la eficiencia técnica de las organizaciones del sector público: aplicación en los equipos de atención primaria", Revista Española de Financiación y Contabilidad, vol. 27, núm. 97, pp. 979-1004, <https://dialnet.unirioja.es/servlet/ articulo?codigo=44285> [consulta: marzo de 2017].

Hernández-González, Rafael, Guadalupe Vadillo-Bueno y Sonia Rivera-Leónides (2008), "Eficacia educativa: avances de un modelo para la educación superior", Magis, Revista Internacional de Investigación Educativa, vol. 1, núm. 1, pp. 63-80.

Johnes, Jill (2006), "Data envelopment analysis and its application to the measurement of efficiency in higher education", Economics of Education Reviere, núm. 3, pp. 273-288. 
Lenin, J. César, Rodrigo Gómez y Zacarías Torres (2016), "Las universidades en México: una medida de su eficiencia a través del análisis de la envolvente de datos con Bootstrap", Acta Universitaria, vol. 26, núm. 6.

López, Janet (2008), "Reseña de Las universidades tecnológicas mexicanas. Un modelo eficaz, una inversión pública exitosa, un sistema a fortalecer, de Jacques Mazeran", Tiempo de Educar, vol. 9, núm. 17, pp. 147-152.

Lucena, Lorena y Gabriel Moreira (2015), "Level of technical efficiency of federal institutes of education, science and technology and the relation between costs, indicators of expansion and retention in efficiency scores", Revista de Educação e Pesquisa em Contabilidade, vol. 9, núm 3, pp. 285-301.

Martín, Raquel (2008), "La medición de la eficiencia universitaria: una aplicación del análisis envolvente de datos", Formación Universitaria, vol. 1, núm. 2, pp. 17-26.

Martínez, Carmen e Isidoro Guzmán (2014), "Medida de la eficiencia en entidades no lucrativas: un estudio empírico para fundaciones asistenciales", Revista de Contabilidad, vol. 17, núm. 1, pp. 47-57.

Martínez, Fidel, J. Carlos de Miguel y Pilar Murias (2005), "El análisis envolvente de datos en la construcción de indicadores sintéticos. Una aplicación a las provincias españolas", Estudios de Economía Aplicada, vol. 23, núm. 3, pp. 753-771.

Martínez, Fidel y Pilar Murias (2011), "Sistemas de pensiones y bienestar económico de la población mayor: un indicador sintético para los países de la OCDE”, Revista Galega de Economía, vol. 20, núm. extraord. 2011, pp. 1-20.

Mazeran, Jacques, John Mallea, Josette Travert, Christian Greuin y Patrice Lafleur (2006), Las universidades tecnológicas mexicanas: Un modelo eficaz, una inversión pública exitosa, un sistema a fortalecer, México, GGUT-SEP.

Modelo de Evaluación de la Calidad del Subsistema de Universidades Tecnológicas (MECASUT) (2014), Base de datos 2014, México, MECASUT, Coordinación General de Universidades Tecnológicas y Politécnicas, <http://cgutyp.sep.gob.mx/Areas/
CoordPGA/DirPlaneaEvalInfo/SubEvaluacion/ MECASUT_2014.xlsx> [consulta: mayo de 2017]. MECASUT (2011), Guía Técnica del MECASUT, México, Coordinación General de Universidades Tecnológicas y Politécnicas, GGUT-A02H-PO-28. L-CPGA-SE-01. MECASUT (2014), Base de datos 2014. Modelo de Evaluación de la Calidad del Subsistema de Universidades Tecnológicas. Coordinación General de Universidades Tecnológicas y Politécnicas, <http://cgutyp.sep.gob.mx/Areas/ CoordPGA/DirPlaneaEvalInfo/SubEvaluacion/ MECASUT_2014.xlsx> [consulta: mayo de 2017].

Nazarko, Joanicjusz y Jonas Saparauskas (2014), "Application of DEA method in efficiency evaluation of public higher education institutions", Technological and Economic development of Economy, vol. 20, núm. 1, pp. 25-44.

Quindós, María del Pilar, Fernando Rubiera y María Rosalía Vicente (2003), "Análisis envolvente de datos: una aplicación al sector de los servicios avanzados a las empresas del Principado de Asturias", Rect@, vol. 21, núm. 1.

Ruiz-Larraguivel, Estela (2011), "La educación superior tecnológica en México. Historia, situación actual y perspectivas", Revista Iberoamericana de Educación Superior, vol. 2, núm. 3, pp. 35-52, <http://www. scielo.org.mx/scielo.php?script $=$ sci_arttext\&pid $=$ S2007-28722011000100002 $>$ [consulta: abril de $2017]$.

Ruiz, Estela (2009), "Los técnicos superiores universitarios. Diferenciación educativa, estratificación social y segmentación del trabajo", Revista Mexicana de Sociología, vol. 71, núm. 3, pp. 557-584.

Silva, Marisol (2008), "Impacto de las políticas de calidad en los procesos educativos de la educación superior", Perfiles Educativos, vol. 30, núm. 120, pp. 7-32, <http:// www.scielo.org.mx/scielo.php?script=sci_arttext\&pi $\mathrm{d}=$ S0185-26982008000200002> [consulta: abril de $2017]$.

Silva, Marisol (2006), La calidad educativa de las universidades tecnológicas. Su relevancia, su proceso de formación y sus resultados, México, ANUIES. 
Stukalina, Yulia (2012), "Addressing service quality issues in higher education: the educational environment evaluation from the students' perspective", Technological and Economic Development of Economy, vol. 18, núm. 1, pp. 84-98.

Valenzuela, Jaime, María Soledad Ramírez y Jorge Alfaro (2009), "Construcción de indicadores institucionales para la mejora de la gestión y la calidad educativa", Revista Iberoamericana de Evaluación Educativa, vol. 2, núm. 2, pp. 59-81.

Villa, Lorenza (2008), "Reseña de La calidad educativa de las universidades tecnológicas. Su relevancia, su proceso de formación y sus resultados, de Marisol Silva", Revista de la Educación Superior, vol. 37, núm. 145, pp. 143-152.

\section{Cómo citar este artículo:}

Aguilar-Barceló, José-Gabriel y Ana-Bárbara Mungaray-Moctezuma (2019), “La empleabilidad de los egresados de las universidades tecnológicas en México: un análisis de eficiencia", en Revista Iberoamericana de Educación Superior (RIES), México, UNAM-IISUE/ Universia, vol. x, Núm. 29, pp. 3-24, Dol: https://doi.org/10.22201/iisue.20072872e.2019.29.520 [consulta: fecha de última consulta]. 